\title{
The retroclavicular approach to the PECS II (RAP) block: a novel regional technique for breast surgery
}

\author{
Kelly M. Martin ${ }^{1}$, Bill Johnson ${ }^{2}$, and Rob R. Taylor ${ }^{2}$ \\ ${ }^{1}$ Department of Anesthesiology, WellSpan Health, York Hospital, York, PA, ${ }^{2}$ Middle Tennessee School of Anesthesia, \\ Madison, TN, USA
}

Goals of perioperative analgesia during breast cancer surgery are to supplement operative anesthesia, reduce acute postoperative pain, and reduce the incidence of post mastectomy pain syndrome (PMPS). Numerous regional anesthesia (RA) techniques (i.e., thoracic paravertebral block [TPVB] and interfascial plane blocks) have been extensively investigated in breast surgery. The PECS II block [1] may have a greater effect on reducing postoperative opioid consumption for the first $24 \mathrm{~h}$ and postoperative pain scores for the first $12 \mathrm{~h}$ compared to that of the TPVB, which is often thought of as the gold standard of RA for breast analgesia [2].

The PECS II block is easy to perform awake or under general anesthesia, is relatively devoid of any serious complications compared to TPVB, and may be better suited for outpatient surgery. The PECS II block is commonly performed as a single injection technique which is its biggest disadvantage because the duration of analgesia is limited to the pharmacokinetics of the local anesthetic (LA) mixture. The TPVB is often performed with a catheter to extend the duration of analgesia for several

Corresponding author: Kelly M. Martin, MS, CRNA

Department of Anesthesiology WellSpan Health, York Hospital, 45

Percheron Dr, York, Pennsylvania 17406, USA

Tel: +1-8142825126, Fax: +1-7178491651

Email: kelly.martin1019@gmail.com

ORCID: https://orcid.org/0000-0003-3511-4764

This work was presented at the 37th Annual ESRA Congress, September 2018, The Convention Centre Dublin, Dublin, Ireland

Received: June 30, 2019.

Revised: July 15, 2019.

Accepted: July 17, 2019.

Korean J Anesthesiol 2019 October 72(5): 512-514

https://doi.org/10.4097/kja.19294 days postoperatively. Blanco et al. [1] described the placement of a catheter in his original description although this is uncommon practice because the PECS catheter lies within the surgical field during most breast surgeries. We describe a novel approach for the preoperative placement of a continuous catheter in the PECS II plane, allowing for preemptive analgesia while preserving the longevity of the block. We have named this novel technique the Retroclavicular Approach to the PECS II (RAP) block.

This technique stems from an earlier description of an ultrasound (US)-guided posterior approach to the infraclavicular brachial plexus [3]. The patient is placed in the supine position with the ipsilateral arm adducted and the head rotated contralaterally to optimize space in the supraclavicular fossa. A linear transducer $(6-15 \mathrm{MHz})$ is used for block placement. Sancheti et al. [4] recently reported the theoretical risk of inadvertent suprascapular nerve (SSN) injury while using a retroclavicular approach. A pre-block scan to locate the SSN in the supraclavicular fossa, inserting the needle lateral and posterior to its location, and utilizing a nerve stimulator while needling to monitor for motor response will reduce this risk.

To perform the RAP block (Figs. 1A-1D), the transducer is placed in the infraclavicular fossa in a parasagittal orientation to identify the axillary vessels, the cords of the brachial plexus, and the pectoralis major and minor muscles (PMM, PmM). A $150-\mathrm{mm}, 18$-gauge Tuohy needle is inserted $2-3 \mathrm{~cm}$ posterior to the clavicle at the superior aspect of the trapezius muscle, medial to the acromioclavicular joint. The needle is advanced into the axillary inlet between the posterior border of the clavicle and the superior border of the scapula. An initial blind zone is encountered on US resulting from the acoustic shadow cast by the clavicle preventing needle visualization. Upon entering the infraclavicular region, the needle can be seen on US and is guided anterior to the axillary vessels towards the interfascial plane

(c) This is an open-access article distributed under the terms of the Creative Commons Attribution Non-Commercial License (http://creativecommons.org/ licenses/by-nc/4.0/), which permits unrestricted non-commercial use, distribution, and reproduction in any medium, provided the original work is properly cited. 

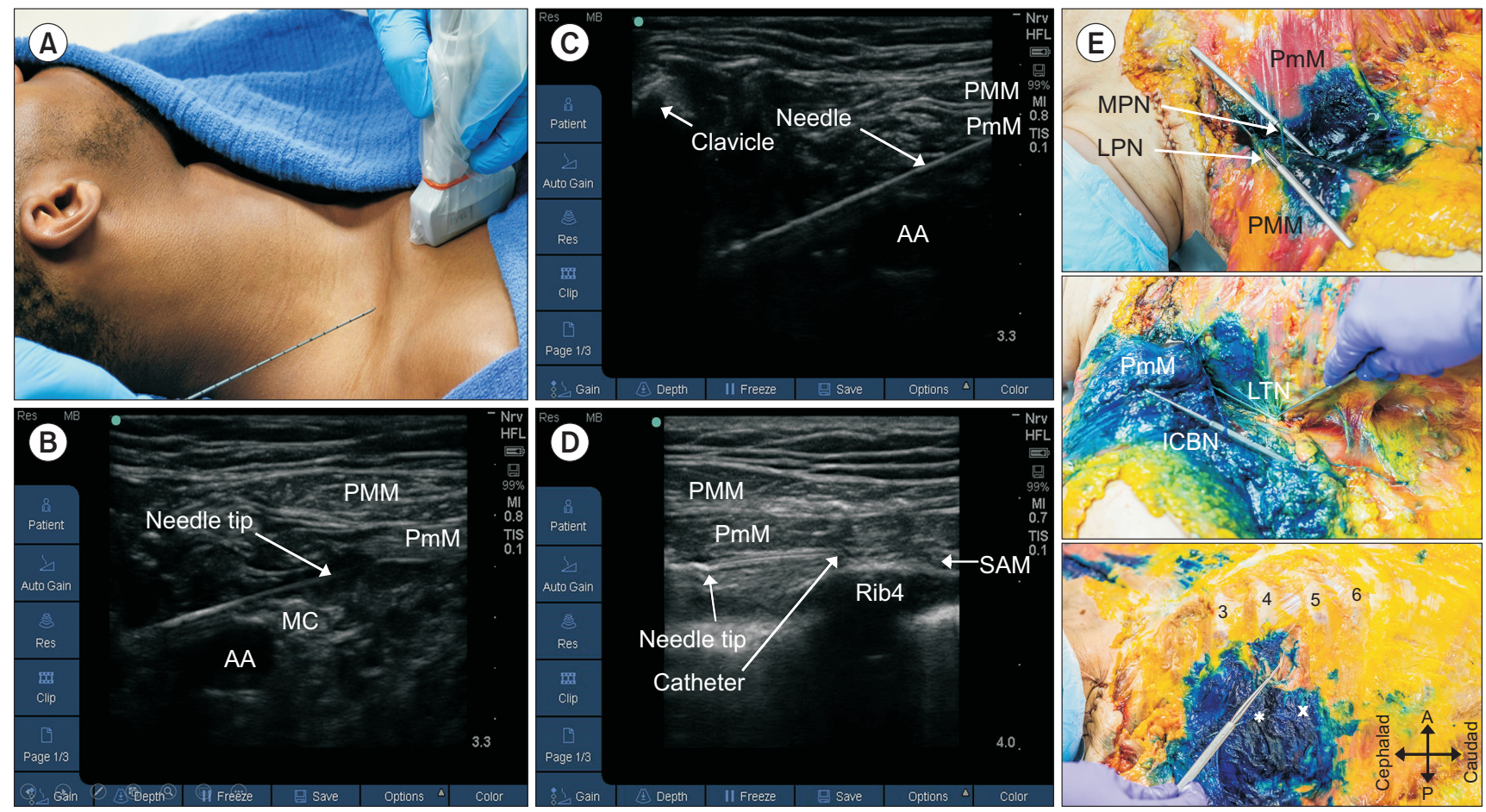

Fig. 1. (A) Needle and probe orientation relative to the clavicle. (B) Needle guided anterior to AA and BP to the fascial plane between PmM and SAM. (C) Acoustic shadow created by the clavicle. Needle seen entering the infraclavicular region and continuing to the PECS II plane. (D) Final position of the catheter seen with the tip above the fourth rib. (E) With the PMM reflected, the LPN and MPN are visualized and are stained with dye. Note the pooling of injectate towards the axilla in this specimen (top). PmM is cut and reflected and the lateral chest wall is visualized. The ICBN and LTN are visualized and are stained with dye (middle). Anterolateral chest wall is exposed with ribs 3-6 labeled. Needle tip $\left({ }^{*}\right)$ and catheter tip (x) identified. Note probe identifying ACB of 4th ICN that is only lightly stained with dye (bottom). AA: axillary artery, BP: brachial plexus, PmM: pectoralis minor muscle, SAM: serratus anterior muscle, PMM: pectoralis major muscle, LPN: lateral pectoral nerve, MPN: medial pectoral nerve, ICBN: intercostobrachial nerve, LTN: long thoracic nerve, ACB: anterior cutaneous branch, ICN: intercostal nerve, MC: medial cord.

between the PmM and serratus anterior muscle at the level of the third rib. Hydrodissection using $20 \mathrm{ml}$ of LA creates a space for catheter placement. The catheter is threaded $3-5 \mathrm{~cm}$ beyond the needle tip and is secured above the clavicle, away from the surgical field.

We assessed the dye spread in two lightly embalmed cadavers using $20 \mathrm{ml}$ of $1 \%$ methylene blue. A catheter was also threaded $3-5 \mathrm{~cm}$ beyond the needle tip after dye injection. The intercostobrachial nerve and lateral cutaneous branches of intercostal nerves 3-6 were heavily stained in both cadavers with spread as low as the seventh intercostal space. The anterior cutaneous branches (ACBs) were assessed at several levels and had only minimal staining (Fig. 1E). It is unclear if this limited spread to the ACBs would result in clinically significant analgesia to the medial aspect of the breast in living subjects. Both cadavers had extensive spread to the lateral chest wall and axilla which resulted in extensive staining of both the long thoracic nerve and thoracodorsal nerve. In one cadaver, spread was noted in the interfascial plane between the pectoralis muscles (Fig. 1E), extensively staining the medial and lateral pectoral nerves; these nerves were almost completely spared in the second cadaver.
The catheters were situated appropriately within the PECS II plane in both cadavers. Further dissection was performed to investigate the needle path and identify any violation of the neurovascular structures. In both cases, the needle successfully passed from the superior aspect of the trapezius muscle, through the axillary inlet, to the PECS II plane without any neurovascular disruption.

\section{Clinical Considerations}

First, the needle must be sufficiently posterior to the clavicle to allow for passage through the axillary inlet and into the infraclavicular space where it then passes anterior to the axillary vessels. Factors that may prohibit needle passage from the retroclavicular space to the PECS plane include: neck circumference and length, highly angulated or anatomical variations of the clavicle, and fullness of the supraclavicular fossa [5]. Elevating the ipsilateral shoulder will increase the supraclavicular space and distance between the posterior border of the clavicle and superior border of the scapula [5]. Secondly, the needle path traverses an area of high vascularity (e.g., axillary vessels, cephalic 
vein, and thoracoacromial artery); therefore, one must strictly follow the American Society of Regional Anesthesia guidelines for anticoagulant status.

We report satisfactory staining of the nerves relevant to breast analgesia and successful catheter placement with this novel approach to the PECS II plane. The RAP block allows for preoperative catheter placement which promotes preemptive analgesia while also preserving the longevity of the block via a continuous LA infusion. This may not only improve acute postoperative pain management but may also reduce the incidence of chronic PMPS. Additional research investigating the safety and efficacy of this novel regional technique is warranted.

\section{Acknowledgments}

The authors thank John Halle and Christi Williams, Department of Physical Therapy, Belmont University, Nashville, Tennessee, for their assistance with cadaveric dissections.

\section{Conflicts of Interest}

No potential conflict of interest relevant to this article was reported.

\section{Author Contributions}

Kelly M. Martin (Conceptualization; Methodology; Writingoriginal draft)

Bill Johnson (Resources; Writing-review \& editing)

Rob R. Taylor (Formal analysis; Supervision; Writing-review \& editing)

\section{ORCID}

Kelly M. Martin, https://orcid.org/0000-0003-3511-4764

Bill Johnson, https://orcid.org/0000-0003-3312-482X

Rob R. Taylor, https://orcid.org/0000-0001-9291-398X

\section{References}

1. Blanco R, Fajardo M, Parras Maldonado T. Ultrasound description of Pecs II (modified Pecs I): a novel approach to breast surgery. Rev Esp Anestesiol Reanim 2012; 59: 470-5.

2. Wahba SS, Kamal SM. Thoracic paravertebral block versus pectoral nerve block for analgesia after breast surgery. Egypt J Anesth 2014; 30 : 129-35.

3. Hebbard P1, Royse C. Ultrasound guided posterior approach to the infraclavicular brachial plexus. Anaesthesia 2007; 62: 539.

4. Sancheti SF, Uppal V, Sandeski R, Kwofie MK, Szerb JJ. A cadaver study investigating structures encountered by the needle during a retroclavicular approach to infraclavicular brachial plexus block. Reg Anesth Pain Med 2018; 43: 752-5.

5. Beh ZY, Hasan MS, Lai HY, Kassim NM, Md Zin SR, Chin KF. Posterior parasagittal in-plane ultrasound-guided infraclavicular brachial plexus block-a case series. BMC Anesthesiol 2015; 15: 105. 